\title{
Diagnostic difficulty arising from rectal recovery in ulcerative colitis
}

\author{
T S Levine, M Tzardi, S Mitchell, C Sowter, A B Price
}

\begin{abstract}
Aims-To ascertain whether the dogma that a normal rectal biopsy precludes a diagnosis of ulcerative colitis is correct. Methods-Rectal biopsy specimens from a prospective group of 24 asymptomatic patients, with an established diagnosis of ulcerative colitis, were examined in a blinded study alongside 10 normal rectal biopsy specimens from an age and sex matched patient cohort without ulcerative colitis. Each biopsy specimen was assessed by three pathologists and ascribed to one of four categories: normal; borderline abnormality (one or more minor nonspecific abnormalities which, when combined, did not fulfil the minimal acceptable criteria for a diagnosis of ulcerative colitis); minimal features of chronic ulcerative colitis; and unequivocal ulcerative colitis.
\end{abstract}

Results-Two patients with ulcerative colitis had normal biopsy specimens; nine specimens were categorised as borderline abnormality, one as showing the minimal changes of chronic ulcerative colitis, and 12 as having the typical changes of chronic ulcerative colitis. Thus, 11 (46\%) of the 24 patients had a rectal biopsy specimen that was devoid of the acceptable attributes on which a diagnosis is established, despite a confident previous diagnosis. Ten of these 11 cases had disease limited to the rectum. Review of all previous histological biopsy specimens $(n=164)$ and clinical data, including drug treatment, failed to identify any attributes that might be prognostic markers for future rectal mucosal healing. Conclusions-A normal rectal biopsy specimen, though uncommon, may occur in longstanding colitis. Moreover, in $46 \%$ of these asymptomatic but established cases the degree of healing may preclude a diagnosis of ulcerative colitis without comprehensive clinical and radiological details. Pathologists need to be aware of this minimal end of the spectrum of disease. (f Clin Pathol 1996;49:319-323)

Keywords: ulcerative colitis, biopsy specimen.

A longstanding guideline for both the clinician and diagnostic histopathologist in the differential diagnosis of chronic inflammatory bowel disease has been that the presence of a normal rectal biopsy specimen can be used to exclude a diagnosis of ulcerative colitis. ${ }^{12}$

The aim of this study was to assess the histological features of ulcerative colitis in rectal biopsy specimens from a patient cohort with longstanding ulcerative colitis who were currently asymptomatic; and to establish from this whether the rectal mucosa could indeed return to normality. We reviewed all the patients' previous clinical, radiological and histological data to try to identify any factor(s) that might influence the natural history of rectal biopsy morphology.

\section{Methods}

Over 18 months patients with ulcerative colitis in clinical remission, attending the routine inflammatory bowel disease outpatient clinic, were enrolled into the study. Remission was defined as normal bowel frequency without blood in the stool for at least one month prior to the current rectal biopsy specimen.

Each patient had an established diagnosis of ulcerative colitis made previously by a combination of clinical, radiological and histological parameters-the latter including at least one previous biopsy specimen with the unequivocal features of ulcerative colitis. ${ }^{3}$ At the outpatient visit a rectal biopsy specimen was taken at least $10 \mathrm{~cm}$ from the anal verge. Subsequently all previous radiological and clinical data were analysed and particular note was made of current and previous drug treatment.

\section{HISTOLOGY}

The rectal biopsy specimen from each patient was routinely fixed in $10 \%$ formalin and embedded in paraffin wax. Three levels were cut at $3 \mu \mathrm{m}$ thickness and stained with haematoxylin and eosin.

Three pathologists independently analysed each biopsy specimen for the characteristic features of ulcerative colitis using the best orientated section(s). The parameters assessed included: crypt atrophy, by estimating crypt shortening and loss; crypt distortion, by the degree of crypt branching and kinking; goblet cell reduction; Paneth cell metaplasia; and the presence of increased numbers of neuroendocrine cells. Chronic inflammatory changes within the lamina propria were assessed according to the degree and distribution of lymphohistiocytic infiltrate, the number of plasma cells present and the presence of basal lymphoid aggregates or follicles. In addition, any apparent hypocellularity of the lamina propria was documented as such, as this can be indicative of long term quiescent ulcerative colitis in a biopsy specimen that would otherwise be regarded as normal. ${ }^{3}$ Activity was defined as increased numbers of neutrophils 
within the lamina propria, including the presence of cryptitis and crypt abscess formation. Each of these variables, except hypocellularity which was scored as present/absent, were graded in severity from none $(0)$ to severe $(+++)$.

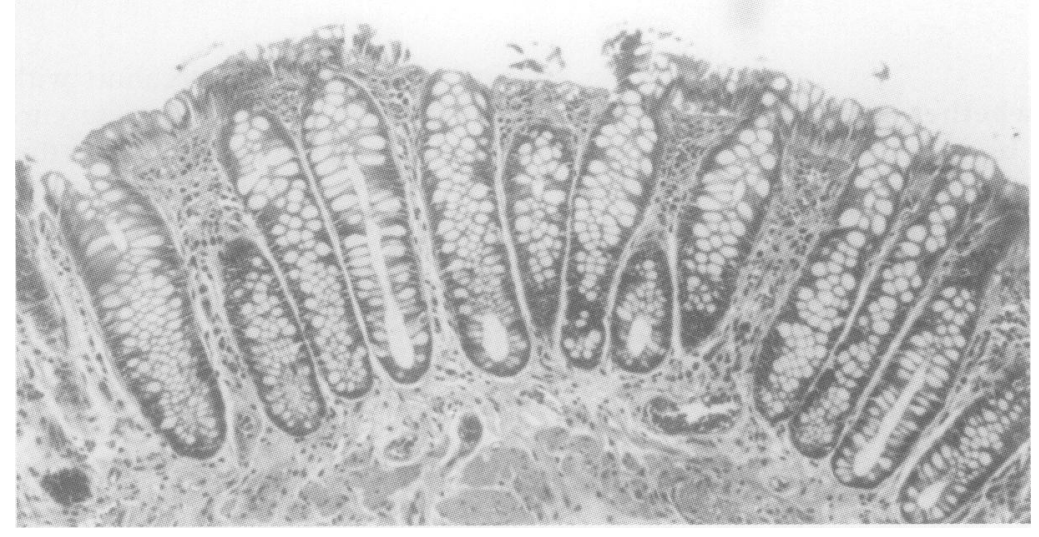

Figure 1 Histologically normal rectal biopsy specimen (patient 1).

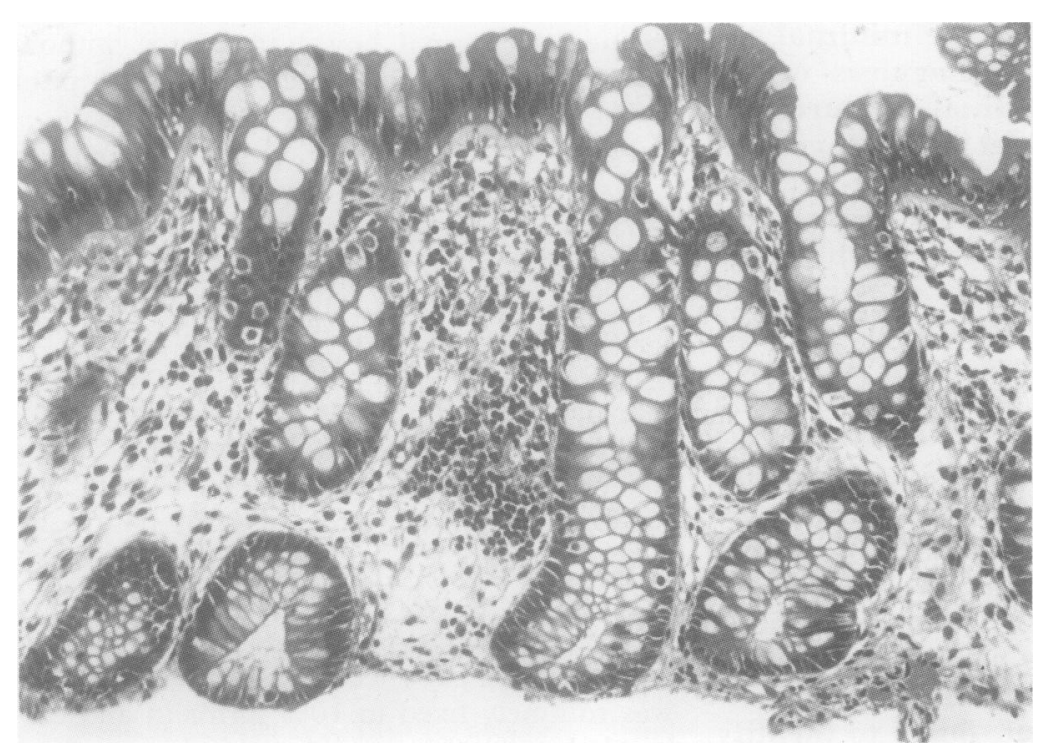

Figure 2 Rectal biopsy specimen with a single small focus of chronic inflammatory cells within the lamina propria (patient 6).

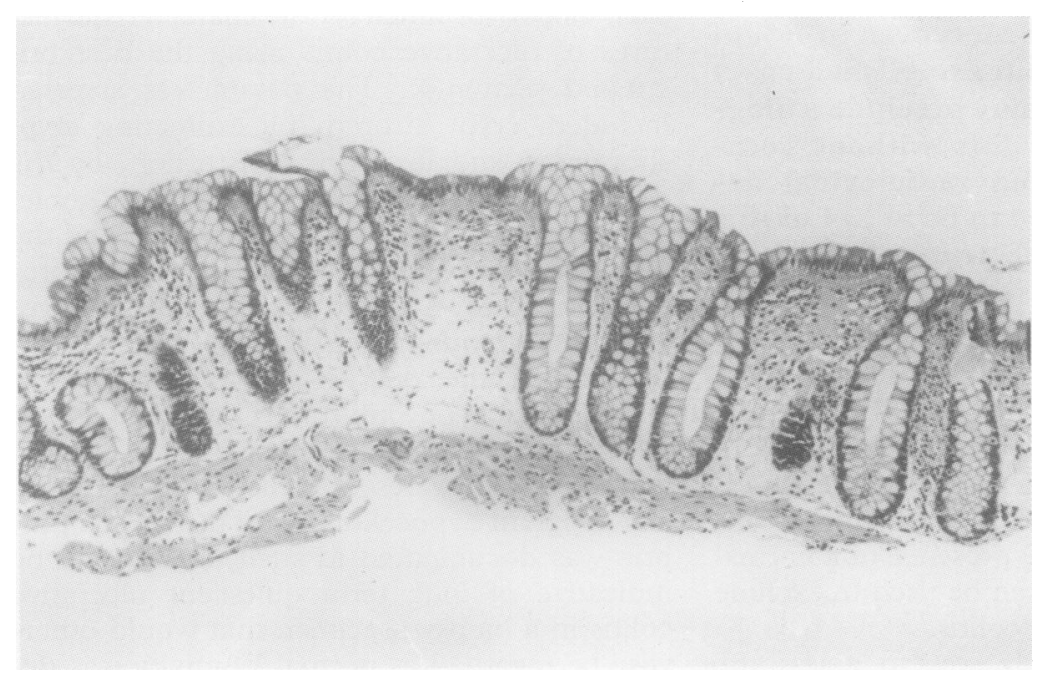

Figure 3 Oedematous lamina propria in a rectal biopsy specimen (patient 4).
Table 1 Histological classification

\begin{tabular}{lcl}
\hline Histological group & $\begin{array}{c}\text { Patients } \\
(n=24)\end{array}$ & $\begin{array}{l}\text { Controls } \\
(n=10)\end{array}$ \\
\hline Normal & 2 & 6 \\
Borderline abnormality & 9 & 4 \\
Minimal ulcerative colitis & 1 & 0 \\
Chronic ulcerative colitis & 12 & 0 \\
\hline
\end{tabular}

Following the analysis each biopsy specimen was assigned to one of four histological groups: (1) normal; (2) borderline abnormality, but non-specific - that is, one or more non-specific architectural or inflammatory abnormalities which, when combined, were not extensive enough to fulfil the minimal criteria for a diagnosis of chronic ulcerative colitis; (3) minimal features of chronic ulcerative colitis, such as hypocellularity and mild atrophy; and (4) typical changes of chronic ulcerative colitis.

In addition to the index rectal biopsy specimen all previous rectal and colonic biopsy specimens from each patient were similarly assessed. Particular attention was paid to the presence/absence of ulceration as it was thought that this might relate to the subsequent ability of the mucosal architecture to recover.

Rectal biopsy specimens from the departmental files, reported as normal, from 10 age and sex matched patients served as controls. These 10 patients had been biopsied while being investigated for irritable bowel syndrome or putative infectious colitis. All had normal rectosigmoidoscopic findings. The assessors were unaware which group each specimen had been assigned to.

\section{Results}

CLINICAL FINDINGS

Twenty four patients (10 men and 14 women; age range $32-88$ years) qualified for the study. Disease duration varied from three to 32 years. All patients were clinically asymptomatic.

Nineteen of the 24 patients also underwent sigmoidoscopy; the rectal mucosa in these patients was normal in nine and showed chronic inactive changes in 10.

HISTOLOGY

Eight of the 34 biopsy specimens assessed (24 from patients and 10 from controls) (table 1) were normal. Two of the eight were from patients with ulcerative colitis (fig 1); the remainder were from controls. Thirteen specimens were classified as borderline abnormality, nine of which were from patients with ulcerative colitis (figs 2 and 3 ) and four from controls. One specimen from a patient with ulcerative colitis was assigned to the minimal ulcerative colitis group (fig 4). The remaining 12 specimens were all from patients with ulcerative colitis and exhibited the histological changes characteristic of chronic disease (figs 5 and 6).

There was $100 \%$ agreement between the assessors in assigning specimens to the normal and chronic ulcerative colitis groups. There was some variation in allocating specimens to the borderline abnormality and minimal 


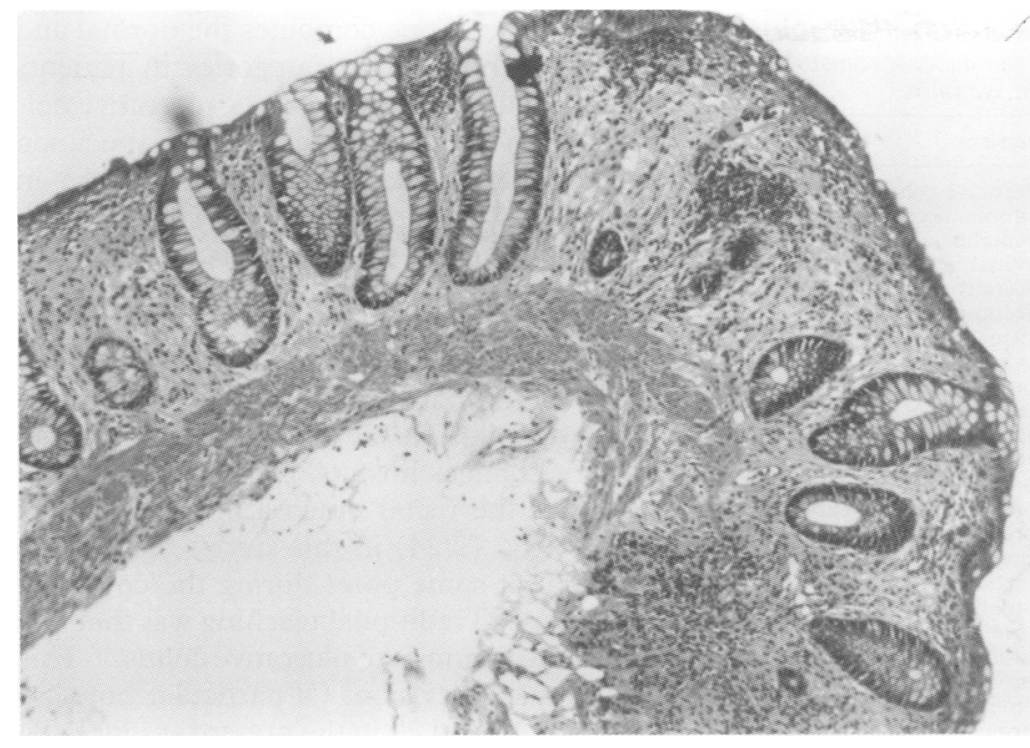

Figure 4 Rectal biopsy specimen with borderline atrophy and hypocellularity of the lamina propria (patient 12).

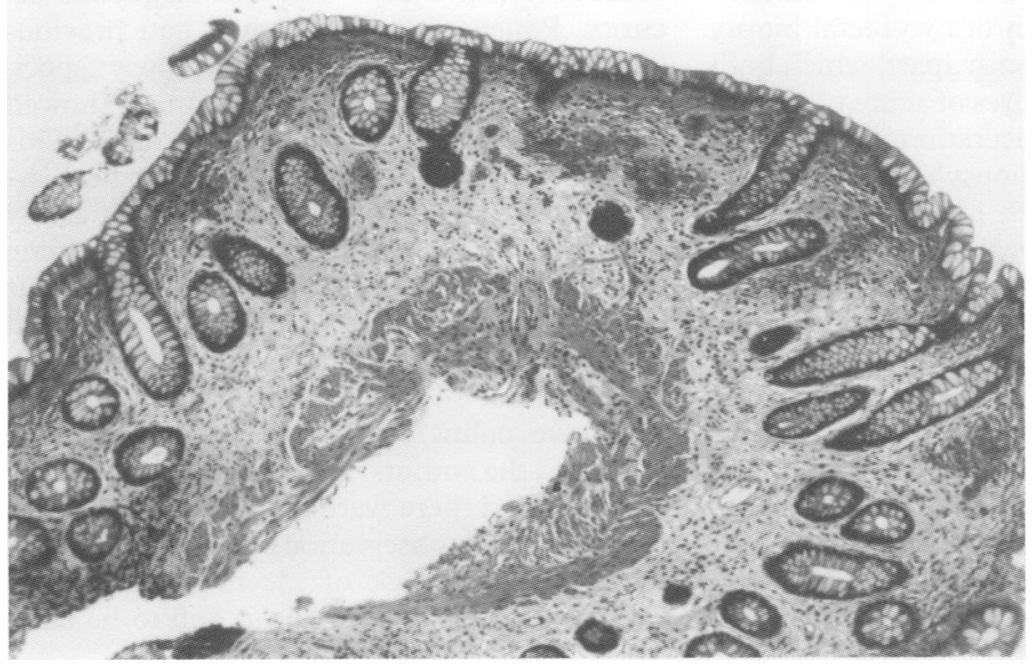

Figure 5 Rectal biopsy specimen with typical changes of inactive chronic ulcerative colitis (patient 14).

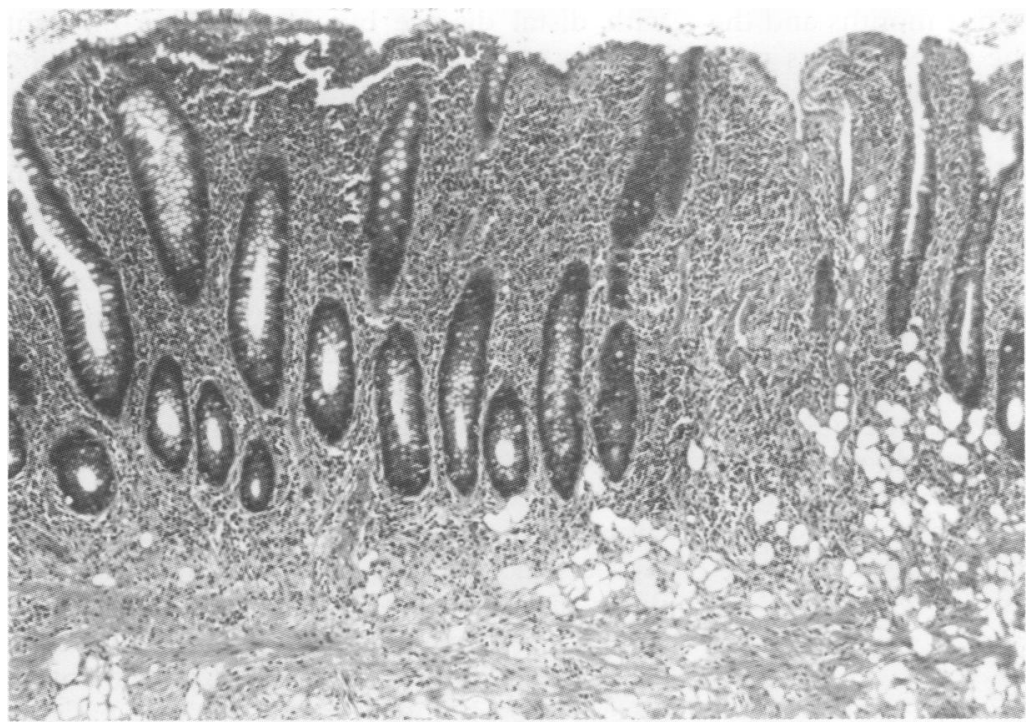

Figure 6 Rectal biopsy specimen with typical changes of chronic active ulcerative colitis (patient 16).
Table $2 A \quad$ Rationale for classification of patients with ulcerative colitis

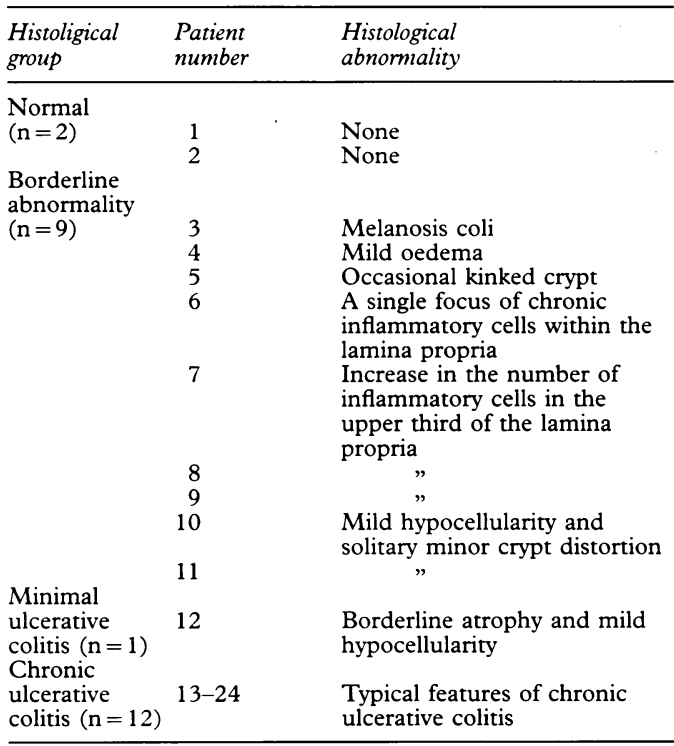

Table 2B Microscopic abnormalities identified in controls

\begin{tabular}{ll}
\hline Histological group & Histological abnormality \\
\hline $\begin{array}{l}\text { Normal }(\mathrm{n}=6) \\
\text { Borderline abnormality } \\
(\mathrm{n}=4)\end{array}$ & $\begin{array}{l}\text { Normal } \\
\text { Oedema } \\
\text { Mild increase in the number } \\
\text { of chronic inflammatory cells } \\
\text { in the upper third of the } \\
\text { lamina propria }\end{array}$ \\
\hline
\end{tabular}

ulcerative colitis subgroups; these discrepancies were resolved by consensus discussion. Tables $2 \mathrm{~A}$ and $2 \mathrm{~B}$ summarise the rationale behind the classification of the ulcerative colitis and control cohorts into the histological subgroups.

Review of the patients' previous histology (164 biopsy specimens in total) revealed that: 16 of the 24 patients had limited ulcerative proctitis; one had left-sided ulcerative colitis; three had subtotal ulcerative colitis (up to the hepatic flexure); and four had pancolitis (table 3). In 10 of the 11 cases currently classified as normal or borderline abnormality, disease was limited to the rectum. By contrast, only one patient of the eight with previous disease proximal to the rectum had a normal or borderline abnormality biopsy specimen.

Four patients had had previous biopsy specimens that on review we classified as normal or borderline abnormality, of which two had current biopsy specimens categorised as normal or borderline abnormality. The biopsy specimen profile of these four patients is shown in table 4 . The remaining nine patients with normal or borderline abnormality biopsy specimens all had unequivocal ulcerative colitis

Table 3 Previous extent of colitis

\begin{tabular}{|c|c|c|c|c|}
\hline $\begin{array}{l}\text { Histological } \\
\text { group }\end{array}$ & $\begin{array}{l}\text { Ulcerative } \\
\text { proctitis }\end{array}$ & $\begin{array}{l}\text { Left-sided } \\
\text { colitis }\end{array}$ & $\begin{array}{l}\text { Subtotal } \\
\text { colitis }\end{array}$ & Pancolitis \\
\hline \multirow{5}{*}{$\begin{array}{l}\text { Normal } \\
\text { Borderline } \\
\text { abnormality } \\
\text { Minimal } \\
\text { ulcerative } \\
\text { colitis } \\
\text { Chronic } \\
\text { ulcerative } \\
\text { colitis }\end{array}$} & 1 & - & - & 1 \\
\hline & 9 & & & \\
\hline & & & & \\
\hline & - & - & 1 & - \\
\hline & 6 & 1 & 2 & 3 \\
\hline
\end{tabular}


Table 4 Biopsy specimen profile of four patients with previous normal or borderline abnormality specimens prior to the classification of the current specimen

\begin{tabular}{lll}
\hline & Year & Diagnosis \\
\hline Patient 1 & 1970 & Ulcerative colitis \\
& 1986 & Chronic ulcerative colitis \\
& 1992 & Borderline abnormality \\
Patient 2 & 1993 & Normal \\
& 1981 & Ulcerative colitis \\
& 1981 & Ulcerative colitis \\
& 1987 & Normal \\
& 1988 & Normal \\
Patient 18 & 1992 & Borderline abnormality \\
& 1992 & Normal \\
& 1974 & Ulcerative colitis \\
& 1976 & Ulcerative colitis \\
& 1988 & Borderline abnormality \\
& 1992 & Borderline abnormality \\
Patient 21 & 1992 & Borderline abnormality \\
& 1977 & Chronic ulcerative colitis \\
& 1978 & Ulcerative colitis \\
& 1980 & Corderline abnormality \\
& 1982 & Chronic ulcerative colitis \\
& 1984 & Chronic ulcerative colitis \\
& 1988 & Borderline abnormality \\
& 1992 & Chronic ulcerative colitis \\
\hline
\end{tabular}

until this time. Of particular note is patient 2 , who had a 13 year history of ulcerative colitis. A diagnosis of ulcerative colitis was reached initially after examination of two rectal biopsy specimens, taken one month apart, which both displayed the classic features of acute ulcerative proctitis with extensive ulceration. Three of the four subsequent rectal biopsy specimens (one taken in 1987 and 1988, and two in 1992), including the current specimen, were normal or borderline abnormality (on the basis of a borderline increase in the number of chronic inflammatory cells present in the lamina propria). Indeed, on the basis of the third normal rectal biopsy specimen all treatment was withdrawn, whereupon the patient had a sudden and severe relapse. The previous biopsy specimens for all the other patients showed consistent features of ulcerative colitis.

Fourteen patients had biopsy evidence of previous rectal mucosal ulceration. Six of these 14 patients subsequently had a normal or borderline abnormality rectal biopsy specimen.

Of the two patients with normal current rectal biopsy specimens, one had not received any form of treatment for five months and the other for six years. Of the nine patients with borderline abnormality biopsy specimens, three had not received treatment for at least two years, five were currently taking oral salazopyrine, and one had been prescribed rectal prednisolone on a pro re nata basis. The patient with minimal ulcerative colitis had not been on treatment for two years. Three of the 12 patients with well established, chronic ulcerative colitis were on oral salazopyrine alone, four were taking oral mesalazine, one was taking salazopyrine and topical steroids (the latter were used three weeks before the current biopsy specimen was taken), one patient was on predsol enemas, and three had not received treatment for four to five years.

\section{Discussion}

Several factors of diagnostic importance emerge from this study. First, although it was limited to 24 patients, if one combines the normal and borderline abnormality categories in patients with an established diagnosis of ulcerative colitis, $46 \%(11 / 24)$ had a rectal biopsy specimen taken during the course of their disease in which there were no features on which to base the diagnosis. In their absence an erroneous diagnosis of Crohn's disease or resolving infection might be suggested. This emphasises the need for detailed knowledge of the clinical history of the patient and access to previous biopsy material. This is especially critical when a patient changes hospital or physician.

Second, the rectal mucosa in a number of patients $(8 \%(2 / 24)$ in this study) may return to normal at some point during the course of their disease. Traditional teaching was that this excluded a diagnosis of ulcerative colitis. ${ }^{12}$ This concept needs revision. Of particular note are patients 2,18 and 21 in the present study (table 4). In patient 2 the rectal biopsy specimen was normal at a time of severe clinical relapse. This pattern of disease has been noted in fulminant colitis $^{45}$ and pathologists need to be aware of this discordance if they are not to mislead clinicians with a false sense of diagnostic security. Patients 18 and 21 had had previous borderline abnormality rectal biopsy specimens, yet their index biopsy specimens showed the typical features of ulcerative colitis. This highlights the lability of the histological architectural signs. These findings significantly diminish the value of some of the longstanding exclusion criteria for the histological diagnosis of ulcerative colitis. Although Dick and Grayson, ${ }^{6}$ during a follow up study in 1967, reported that 14 of 62 patients with quiescent ulcerative colitis had normal rectal mucosal histology, the authors' criteria of normality were not clear and there were no photomicrographs to support their observations. As yet, the dogma that the first attack of ulcerative colitis invariably starts in the rectum is still to be challenged.

Third, the restoration of normality, or at least minimal, non-specific abnormality, is largely confined to patients with distal disease. In the present study this occurred in 10 of 16 patients with distal disease but in only one of eight patients with extensive disease. This finding needs to be explored further in a larger series.

There are several reviews describing the normal appearances of the rectal mucosa which for diagnostic histopathologists remains a qualitative assessment. ${ }^{7}$ For this reason we did not incorporate quantitative techniques into the study, restricting its scope to the common diagnostic situation. In general, the diagnostic histopathologist does not have the time nor the equipment necessary for morphometric analysis.

Drug treatment, in particular predsol enemas, ${ }^{8}$ is a recognised cause of rectal sparing and consequently may result in a mistaken diagnosis of Crohn's disease. Histology in such cases, however, is seldom entirely normal. Zaitoun $e t a l^{9}$ described an improvement in several morphometric parameters in patients with ulcerative colitis treated with a combination of oral salazopyrine and rectal steroids. 
Interestingly, although epithelial measurements of atrophy improved and numbers of acute and chronic inflammatory cells decreased on this regimen, these parameters did not return to normal when compared with controls. Odze et $a l^{10}$ described the "complete absence" of any of the characteristic features of ulcerative colitis in $50 \%$ of patients treated with topical 5 -aminosalicylic acid. However, their definition of "normal" was based merely upon the absence of the characteristic features of chronic ulcerative colitis.

In the present study five of 11 patients who had normal or borderline abnormality current biopsy specimens had not been on treatment from five months to six years prior to sampling. Furthermore, only one patient had been prescribed rectal steroids; this was on a pro re nata basis for symptoms and the patient had been asymptomatic for several months before sampling. Six patients were on oral salazopyrine only. Thus, even if topical drug treatment "normalises" rectal mucosal histology, this effect was not a factor in our patients.

On reviewing the previous histology it could be hypothesised that crypt atrophy and architectural distortion represent imperfect mucosal healing after bouts of colitis characterised by surface and crypt ulceration. The corollary is that those patients in whom the rectal biopsy specimen had returned to normal might be those in whom ulceration had not been a feature of previous attacks. However, six of 11 patients with a normal or borderline abnormality current biopsy specimen had had epithelial ulceration in previous rectal biopsy specimens. This compares with seven of the 13 patients currently classified as having minimal or chronic ulcerative colitis. We were unable to identify any specific histological feature(s) that influenced subsequent mucosal recovery.

It is interesting that of the four patients who had previous rectal biopsy specimens classified as normal or borderline abnormality, two were from patients whose current rectal biopsy specimens showed unequivocal features of chronic ulcerative colitis. Thus, although the rectal mucosa may normalise, the subsequent histo- logical picture is not static and the histology may deteriorate with relapse. Although, as seen in patient 2 , a relapse need not invariably involve the rectum.

Given the observation that four (40\%) of 10 rectal biopsy specimens from the control group were re-categorised as abnormal, the study underlines that these very minor abnormalities, or even a normal biopsy specimen, can be a feature not only of those without colitis but also of patients with longstanding ulcerative colitis, in particular those with distal disease. Therefore, we suggest that pathologists document any histological abnormalities descriptively, no matter how minor, concluding that such features are "unlikely to be clinically significant in the absence of any other evidence of inflammatory bowel disease".

In summary, a normal rectal biopsy specimen does not exclude a diagnosis of ulcerative colitis and a patient may still be symptomatic despite normal rectal mucosal histology. These findings bring a note of caution to some established concepts of the histological interpretation of chronic ulcerative colitis.

1 Morson BC. The rectal biopsy in inflammatory bowel disease. $N$ Engl f Med 1972;287:1337-9.

2 Podolsky DK. Inflammatory bowel disease. $N$ Engl f Med 1991;325:928-1016.

3 Talbot IC, Price AB. Biopsy pathology in colorectal disease. London: Chapman and Hall, 1987:118-27.

4 Lennard-Jones JE, Vivian AB. Fulminating ulcerative colitis-recent experience and management. BMF 1960;ii: 96.

5 Burnham WR, Ansell ID, Langman MJ. Normal sigmoidoscopic findings in severe ulcerative colitis, an important and common occurrence (abstract). Gut 1980;21:

Dick AP, Grayson MJ. Ulcerative colitis: A follow-up investigation with mucosal biopsy studies: BMF 1967;160: $160-5$.

7 Levine DS, Haggitt RC. Normal histology of the colon. Am f Surg Pathol 1989;13:966-84.

8 Danielsson A, Hellers G, Lyrenas E, Lofberg R, Nilsson A, Olsson $\mathrm{O}$, et al. A controlled randomised trial of budesonide versus prednisolone retention enemas in active distal ulcerative colitis. Scand $\mathcal{f}$ Gastroenterol 1987;22: 87-92.

9 Zaitoun AM, Cobden I, Al Mardim H, Record CO. Morphometric studies in rectal biopsy specimens from patients with ulcerative colitis; effect of oral 5-ASA and rectal prednisolone treatment. Gut 1991;32:183-7.

10 Odze R, Antonioli D, Peppercorn M, Goldman H. Effect of topical 5-aminosalicylic acid (5-ASA) treatment on rectal mucosal biopsy morphology in chronic ulcerative colitis. Am f Surg Pathol 1993;17:869-75. 